\title{
The long flow to freedom
}

\author{
Ofer Aharony, ${ }^{a}$ Shlomo S. Razamat, ${ }^{b}$ Nathan Seiberg ${ }^{c}$ and Brian Willett ${ }^{d}$ \\ ${ }^{a}$ Department of Particle Physics and Astrophysics, Weizmann Institute of Science, \\ Rehovot 7610001, Israel \\ ${ }^{b}$ Department of Physics, Technion, \\ Haifa, 32000, Israel \\ ${ }^{c}$ School of Natural Sciences, Institute for Advanced Study, \\ Princeton, NJ 08540, U.S.A. \\ ${ }^{d}$ Kavli Institute for Theoretical Physics, University of California, \\ Santa Barbara, CA 93106, U.S.A. \\ E-mail: ofer.aharony@weizmann.ac.il, razamat@physics.technion.ac.il, \\ seiberg@ias.edu, bwillett@kitp.ucsb.edu
}

ABstract: Two-dimensional field theories do not have a moduli space of vacua. Instead, it is common that their low-energy behavior is a sigma model with a target space. When this target space is compact its renormalization group flow is standard. When it is noncompact the continuous spectrum of operators can change the qualitative behavior. Here we discuss two-dimensional gauge theories with $\mathcal{N}=(2,2)$ supersymmetry. We focus on two specific theories, for which we argue that they flow to free chiral multiplets at low energies: the $\mathrm{U}(1)$ gauge theory with one flavor (two chiral superfields with charges plus and minus one) and a non-zero Fayet-Iliopoulos term, and pure $\mathrm{SU}(N)$ gauge theories. We argue that the renormalization group flow of these theories has an interesting order of limits issue. Holding the position on the target space fixed, the space flattens out under the renormalization group. On the other hand, if we first go to infinity on the target space and then perform the renormalization group, we always have a non-trivial space, e.g. a cone with a deficit angle. We explain how to interpret low-energy dualities between theories with non-compact target spaces. We expect a similar qualitative behavior also for other non-compact sigma models, even when they do not flow to free theories.

KEYWORDS: Renormalization Group, Supersymmetric gauge theory, Supersymmetry and Duality

ARXiv EPrint: 1611.02763 


\section{Contents}

1 Introduction and summary 1

$2 \mathrm{U}(1)$ with one flavor $\quad 4$

2.1 The Kähler potential and its one-loop renormalization group flow 5

$\begin{array}{lll}3 & \text { Pure } \mathrm{SU}(N) \text { gauge theories } & 8\end{array}$

\section{Introduction and summary}

One characteristic feature of supersymmetric field theories is the existence of flat directions in the classical potential, which we will denote by $\mathcal{M}_{0}$. It is often the case that these lead in the quantum theory to a moduli space $\mathcal{M}$ of inequivalent vacua. The study of this space is one of the main tools in the analysis of these theories. $\mathcal{M}$ is typically non-compact, and because of asymptotic freedom its asymptotic behavior is similar to that of the classical space $\mathcal{M}_{0}$.

This situation changes in two dimensions. Here there is no moduli space of vacua. ${ }^{1}$ Instead, the fluctuations of the quantum theory explore all of $\mathcal{M}$ and it can be viewed as a target space of the low energy excitations. Moreover, the metric of $\mathcal{M}$ contains operators that are classically marginal, and that are crucial for understanding the quantum behavior of the theory, unlike in higher dimensions where only the complex structure of $\mathcal{M}$ is important.

This fact has many interesting consequences. One of them is a puzzle associated with duality. Consider first an IR duality between two different higher dimensional theories $A$ and $B$. Classically, each of them has its own space of flat directions $\mathcal{M}_{0 A}$ and $\mathcal{M}_{0 B}$, which become in the quantum theory $\mathcal{M}_{A}$ and $\mathcal{M}_{B}$. Since typically $\mathcal{M}_{0 A}$ and $\mathcal{M}_{0 B}$ have different asymptotic metrics, and these are the same as those of $\mathcal{M}_{A}$ and $\mathcal{M}_{B}$ respectively, the asymptotic behaviors of $\mathcal{M}_{A}$ and $\mathcal{M}_{B}$ are different. This does not contradict the IR duality between them, because the statement about the duality is only about the IR behavior. The metric at a generic point on these spaces flows to a trivial flat metric in the IR. And the duality simply means that at least one region in $\mathcal{M}_{A}$ is similar to one region in $\mathcal{M}_{B}$.

This cannot be the case in two dimensions. First, as we said above, the quantum theory explores the whole target space $\mathcal{M}$ and we cannot simply focus on one region. Second, unlike the situation in the higher dimensional theory, the deviation of the metric on $\mathcal{M}$ from the flat metric can be relevant or irrelevant. Thus, the metric on $\mathcal{M}(\tau)$ depends nontrivially on the renormalization group parameter $\tau$. However, operators that change the

\footnotetext{
${ }^{1}$ In [1] we will review and discuss situations in which the Hilbert space of the quantum two-dimensional theory splits into a direct sum of Hilbert spaces, each being a separate superselection sector. But even in these cases we do not obtain a continuum of different low-energy theories.
} 
asymptotic metric are not normalizable. Therefore, one might conclude that two different two-dimensional theories $A$ and $B$ with different asymptotic metrics in $\mathcal{M}_{0 A}$ and $\mathcal{M}_{0 B}$ cannot possibly be dual to each other.

One of the points of this paper is that this conclusion could be too fast. The reasoning in the previous paragraph involves two different limits: the IR limit $\tau \rightarrow \infty$ and going to the asymptotic region of $\mathcal{M}(\tau)$. We will argue that often these two limits do not commute. When we discuss IR dualities in two dimensions we must take the IR limit $\tau \rightarrow \infty$ before we go to infinity in $\mathcal{M}(\tau)$. Being careful about this order of limits will allow us to clarify the status of various dualities. For any finite renormalization group time, the metric at infinity can be different in IR-dual theories. However, the metric at fixed positions in the interior of the space, and correlation functions of operators that are localized there, converge to the same values in IR-dual theories as we go to low energies.

Starting with [2], there is a huge body of work analyzing $\mathcal{N}=(2,2)$ supersymmetric field theories and their dynamics. In this paper we will discuss two simple examples of such theories, which are conjectured (based on the comparison of objects protected by supersymmetry) to flow to free theories at low energies. More precisely, the gauge theory first flows to a non-linear sigma model with some metric $\mathcal{M}_{0}$, and then flows to the sigma model on $\mathcal{M}(\tau)$ as above. Further evidence for these dualities comes from reducing three dimensional dualities on a circle, as will be discussed in [1]. In section 2 we will discuss the Higgs branch of a U(1) gauge theory with two chiral superfields $Q$ and $\tilde{Q}$ with charges \pm 1 . In section 3 we will discuss the pure gauge $\mathrm{SU}(N)$ theories. We expect the same general description of the renormalization group flows of non-compact sigma models, and of IR dualities between theories with non-compact moduli spaces, to arise also in interacting examples, such as the gauged linear sigma model for the non-compact conifold. Closely related behavior occurs also in the gauged linear sigma model for the two dimensional black hole [3]. Some additional examples will be analyzed in [4], including examples appearing in IR dualities discussed in $[5-8,1]$.

The Abelian theory we discuss in section 2 can have a Fayet-Iliopoulos (FI) parameter $r$, and we will mostly be interested in non-zero $r$. In 1 (a) we see the classical $\mathcal{M}_{0}$ with $r=0$, which is the cone $\mathbb{C} / \mathbb{Z}_{2}$. (We suppress the Coulomb branch, which emanates from the singular point.) In 1 (b) we see $\mathcal{M}_{0}$ for non-zero $r$, which is a capped cone. We will argue that in the quantum theory $\mathcal{M}(\tau)$ with non-zero $r$ is flattened out as in $1(\mathrm{c})$. The curvature is pushed to infinity and the space becomes flat, without changing the asymptotic behavior. This way for any finite renormalization group (RG) time $\tau \mathcal{M}(\tau)$ has the same asymptotic behavior as $\mathcal{M}_{0}$, but as $\tau \rightarrow \infty \mathcal{M}(\tau)$ becomes flat. This makes it possible for this theory to be dual to a free field theory, whose asymptotic target space is $\mathbb{C}$ rather than $\mathbb{C} / \mathbb{Z}_{2}$. This is a special case of general IR dualities for $\mathrm{U}(N)$ gauge groups, that were tested by the comparison of supersymmetric partition functions in $[9,10]$.

In section 3 we discuss the pure $\mathrm{SU}(N)$ gauge theory. In the simplest case of $\mathrm{SU}(2)$ its classical Coulomb branch is again a cone $\mathcal{M}_{0}=\mathbb{C} / \mathbb{Z}_{2}$ (see figure $2(\mathrm{a})$ ). We argue that its singularity is smoothed out by the renormalization group flow to figure $2(\mathrm{~b})$, such that it is dual (in the sense discussed above) to a free field theory, whose target space is $\mathbb{C}$. Similarly we argue that for all $N$ the theory is dual to $(N-1)$ free chiral multiplets. A similar claim 


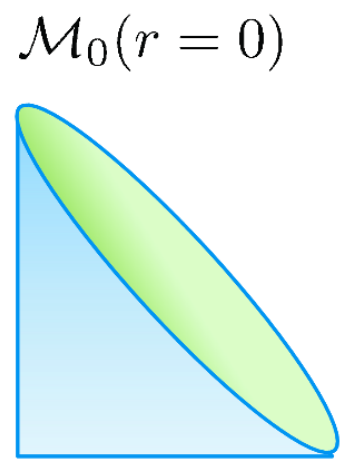

$a$
$\mathcal{M}_{0}(r \neq 0)$

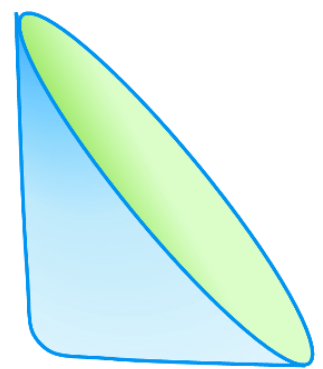

$b$
$\mathcal{M}(r \neq 0, \tau)$

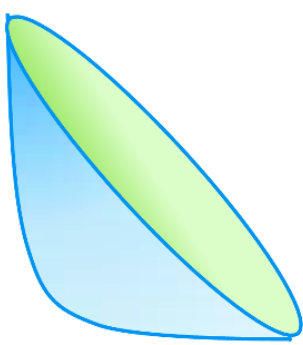

$C$

Figure 1. The Higgs branch of $\mathcal{N}=(2,2)$ SUSY QED. Figure $a$ depicts the classical Higgs branch $\mathcal{M}_{0}(r=0)$ when the FI-parameter $r$ vanishes. Figure $b$ depicts the same space $\mathcal{M}_{0}$ when the FI-term is non-zero. It has the same asymptotic behavior as $\mathcal{M}_{0}(r=0)$, but it is smooth. Figure $c$ shows the effect of the renormalization group flow on $\mathcal{M}(\tau)$. The asymptotic behavior of the space is not modified, but the metric is flattened out. Hence the IR-dual theory is free.

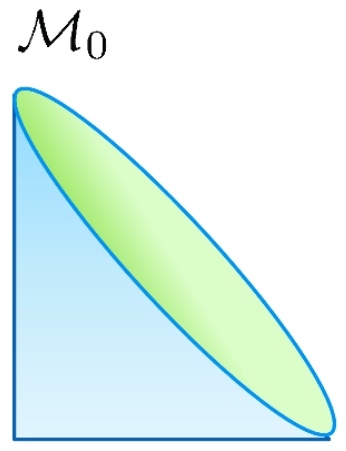

$a$

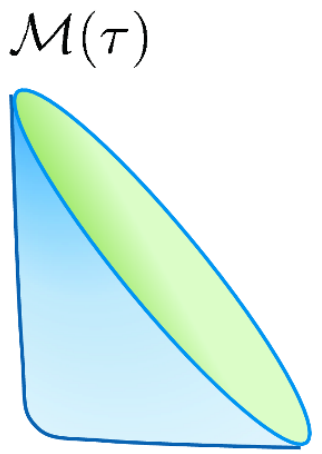

$b$

Figure 2. The moduli space of $\mathcal{N}=(2,2)$ SUSY Yang-Mills theory with gauge group SU(2). Figure $a$ depicts the classical Coulomb branch $\mathcal{M}_{0}$. We conjecture that in the quantum theory it is smoothed out by the renormalization group flow, and $\mathcal{M}(\tau)$ is depicted in figure $b$. The IR dual theory is free.

was made in [5] for $\mathrm{SU}(N)$ gauge theories with $N+1$ chiral multiplets in the fundamental representation.

Similar renormalization group flows in non-linear sigma models were analyzed from a different angle in [11] (some interesting followup papers are [12-15]). They were mostly interested in the use of these theories as worldsheet actions in string theory and in their space-time implications. One-loop renormalization group flows in non-compact non-linear sigma models were also analyzed in [16-18]. In this paper we are only interested in the two dimensional field theory and in the interpretation of low-energy dualities between gauge theories, and we focus on the change in the metric after long renormalization group time. 


\section{$2 \mathrm{U}(1)$ with one flavor}

Our main example will be a U(1) gauge theory with one flavor, i.e., a pair of chiral multiplets with charges \pm 1 . The action of this theory can be written in superspace as:

$$
S=\int d^{2} x \int d^{4} \theta\left(\frac{1}{e^{2}}|\Sigma|^{2}+Q^{\dagger} e^{V} Q+\tilde{Q}^{\dagger} e^{-V} \tilde{Q}-t V\right)
$$

Here $e$ is the gauge coupling, with dimension one, and $t=r+i \theta$ is the complexified FayetIliopoulos (FI) parameter, containing the ordinary FI parameter, $r$, and the theta angle, $\theta$. In the gauge theory $t$, which is classically marginal, does not flow under the RG. However, the low-energy theory has many other classically marginal operators, and we will see that they do flow non-trivially under the RG.

In addition to R-symmetries, this theory has a single $\mathrm{U}(1)_{a}$ global symmetry, under which both chiral multiplets transform with charge 1 . One can weakly gauge this symmetry and turn on a background value for the complex scalar in the gauge multiplet; such a "twisted mass" deforms the theory to a massive theory with a single supersymmetric vacuum for any value of the FI parameter. However, for most of what follows, we will work at vanishing twisted mass, and then we expect the theory defined by the action (2.1) to flow to a superconformal field theory at low energies.

At energies below the photon mass, proportional to the gauge coupling $e$, the photon can be integrated out and the theory can be described in terms of a non-linear sigma model, whose target space $\mathcal{M}_{0}$ can be obtained by solving the $D$-term equations modulo gauge symmetry:

$$
\mathcal{M}_{0}=\left\{|Q|^{2}-|\tilde{Q}|^{2}-r=0\right\} / \mathrm{U}(1)_{\text {gauge }} .
$$

This space is topologically a complex plane, with a natural gauge-invariant chiral coordinate $M \equiv Q \tilde{Q}$. The metric on this space does not receive corrections depending on $e$, but as a non-linear sigma model $\mathcal{M}(\tau)$ flows non-trivially.

We will describe the geometry of this space in more detail below. For $r$ non-zero, it is smooth, while for $r=0$ it has a conical singularity at $M=0$, where the photon is classically massless. If the theta angle is also tuned appropriately, ${ }^{2}$ we expect a singularity in the CFT, associated to the presence of a Coulomb branch emanating from the point $M=0$, on which $\Sigma$ takes non-zero values. This theory is expected to have a continuous spectrum related to a "throat" that develops near the origin and connects (for finite RG time) the Higgs branch to the Coulomb branch, and we will not discuss it further here.

In the remainder of this section we will provide evidence that this theory, for non-zero $r$, is IR-dual to a free chiral multiplet, which can be identified with $M=Q \tilde{Q}$. This duality is a special case of a general $\mathrm{U}(N)$ duality proposed in [9]. It is not expected to hold for the special value $t=i \pi$, for which we do not expect the RG flow to lift the continuum of states living near the origin. But we conjecture that it holds whenever $r \neq 0$.

\footnotetext{
${ }^{2}$ Specifically, this singularity occurs at $\theta=\pi$, rather than $\theta=0$ as one might naively expect, because the effective theta angle at low energies gets a contribution of $\pi$ relative to the bare theta angle after integrating out the chiral multiplets.
} 
The supersymmetric partition functions of the $\mathrm{U}(1)$ gauge theory and the free theory, on both $\mathbb{S}^{2}$ and $T^{2}$, have been shown to agree in [9-19]. The precise relations one obtains from equation (2.45) in [9], and from section 4.6.1 of [10], are:

$$
\mathcal{Z}_{\mathrm{U}(1) N_{f}=1}^{\mathbb{S}^{2}}(\mu, t, \bar{\mu}, \bar{t})=e^{\tilde{W}_{B G}(\mu, t)-c . c \cdot} \mathcal{Z}_{\text {chiral }}^{\mathbb{S}^{2}}(2 \mu, 2 \bar{\mu}), \quad \mathcal{Z}_{\mathrm{U}(1) N_{f}=1}^{T^{2}}(q, y, \nu)=\mathcal{Z}_{\text {chiral }}^{T^{2}}\left(q, y, \nu^{2}\right),
$$

for appropriately defined partition functions that can be computed by localization $[7,8,19]$. In the $T^{2}$ partition function, $q=e^{2 \pi i \tau}$ is the modular parameter, and $y$ and $\nu$ are fugacities for the $\mathrm{U}(1)_{R}$ and $\mathrm{U}(1)_{a}$ symmetries, respectively. The $\mathbb{S}^{2}$ partition function agrees up to a certain contact term, namely, a twisted superpotential depending on the background fields:

$$
\tilde{W}_{B G}(\mu, t)=-2 \mu \log \left(2 \cosh \left(\frac{t}{2}\right)\right)
$$

where $\mu$ is the scalar in the background gauge multiplet coupled to the $\mathrm{U}(1)_{a}$ flavor symmetry. Note that this term diverges at $t=i \pi$, related to the extra light degrees of freedom there, and indicating that the duality fails at this point.

\subsection{The Kähler potential and its one-loop renormalization group flow}

The partition function checks of this duality are quite non-trivial, but they suffer from the basic shortcoming that they are only sensitive to protected information in the theory. For the theories to be precisely dual, they must agree not only in their protected data, but also in unprotected data that affects the low-energy effective action, such as the Kähler potential for $M$. Precisely because such data is not protected, it is difficult to check that it matches across a putative duality. Moreover, as we mentioned in the introduction and will explain in more detail below, the fact that the asymptotic behavior of the target space seems different poses a challenge to the duality. However, at least in some limit of parameters, we will find evidence that the Kähler potential does match across the duality.

Recall that at energies far below the gauge coupling $e$, the gauge field (which is massive on the Higgs branch for generic values of $r$ ) can be integrated out to find an approximate description as a non-linear sigma model into the space (2.2). Let us start by computing the tree-level Kähler potential for the gauge theory. In the limit $e^{2} \rightarrow \infty$, the gauge kinetic term can be ignored, and the Kähler potential is given by:

$$
K=Q^{\dagger} e^{V} Q+\tilde{Q}^{\dagger} e^{-V} \tilde{Q}-r V
$$

The $D$-term constraint on the chiral multiplets is:

$$
Q^{\dagger} e^{V} Q-\tilde{Q}^{\dagger} e^{-V} \tilde{Q}-r=0 .
$$

To solve this, let us write $X=Q^{\dagger} e^{V} Q$ and $\tilde{X}=\tilde{Q}^{\dagger} e^{-V} \tilde{Q}$, and note:

$$
X \tilde{X}=|M|^{2}, \quad X-\tilde{X}-r=0 .
$$

These can be solved for $X$ and $\tilde{X}$ in terms of $|M|^{2}$ and $r$, and we find:

$$
K=\sqrt{r^{2}+4|M|^{2}}-|r| \log \left(|r|+\sqrt{r^{2}+4|M|^{2}}\right) .
$$


This leads to a tree-level metric:

$$
d s^{2}=\frac{1}{\sqrt{r^{2}+4|M|^{2}}} d M d \bar{M} .
$$

Let us first note a few properties of this metric on $\mathcal{M}_{0}$, depicted in figures 1 (a) and 1(b). For $|M| \gg|r|$, the metric behaves as:

$$
d s^{2} \approx \frac{1}{2|M|} d M d \bar{M},
$$

which is flat, but with a deficit angle of $\pi$ at infinity. As $r \rightarrow 0$, the metric approaches that of a cone, $\mathbb{C} / \mathbb{Z}_{2}$, which is flat everywhere except for a conical singularity at $M=0$. For finite $r$ the tip of the cone is smoothed, with curvature of order $1 /|r|$ (going to zero as $M \rightarrow \infty)$. As $|r| \rightarrow \infty$, the space becomes locally flat.

Note from (2.10) that the asymptotic behavior of the metric is very different from that of the putative dual, which is given by the flat metric, $d s^{2}=d M d \bar{M}$. On the other hand, (2.10) is asymptotically flat, so one does not expect it to undergo renormalization group flow. This leads to an apparent contradiction with the proposed duality.

To resolve it, we study the RG flow of the metric. This is governed by the equation:

$$
\partial_{\tau} g_{i \bar{j}}=-\frac{1}{2 \pi} R_{i \bar{j}}+O\left(R^{2}\right)
$$

where $R_{i \bar{j}}$ is the Ricci tensor, and $\tau$ is the RG time. We expect the first term of this equation to give a good approximation to the RG flow when the curvature is everywhere small. This holds for (2.9) provided we take $|r|$ sufficiently large. If we write $M=e^{u+i \theta}$, for $u \in \mathbb{R}$, then (2.9) takes the form:

$$
d s^{2}=\Omega(u)\left(d u^{2}+d \theta^{2}\right),
$$

with:

$$
\Omega(u)=\frac{1}{|r|} \frac{e^{2 u}}{\sqrt{1+\frac{4}{r^{2}} e^{2 u}}} .
$$

Then the one-loop approximation to the RG equation (2.11) becomes:

$$
\partial_{\tau} \Omega(u, \tau)=\frac{1}{4 \pi} \frac{\partial^{2}}{\partial u^{2}}(\log (\Omega(u, \tau)))
$$

with initial condition given by (2.13). Note that redefining $u \rightarrow u+\log (|r| / 2)$ and $\tau \rightarrow|r| \tau$ eliminates $r$ from this equation. Thus the one-loop RG equation is essentially independent of $r$, and so we can set $r=2$ for simplicity. Corrections to (2.14) are proportional to powers of $1 /|r|$.

We solve (2.14) numerically using Mathematica The results are shown in figure 3 and figure 4, for RG times up to $\tau=300$. Here we have used an RG-time-dependent coordinate transformation to place the metric in the form:

$$
d s^{2}=d v^{2}+\Omega(v) d \theta^{2} .
$$




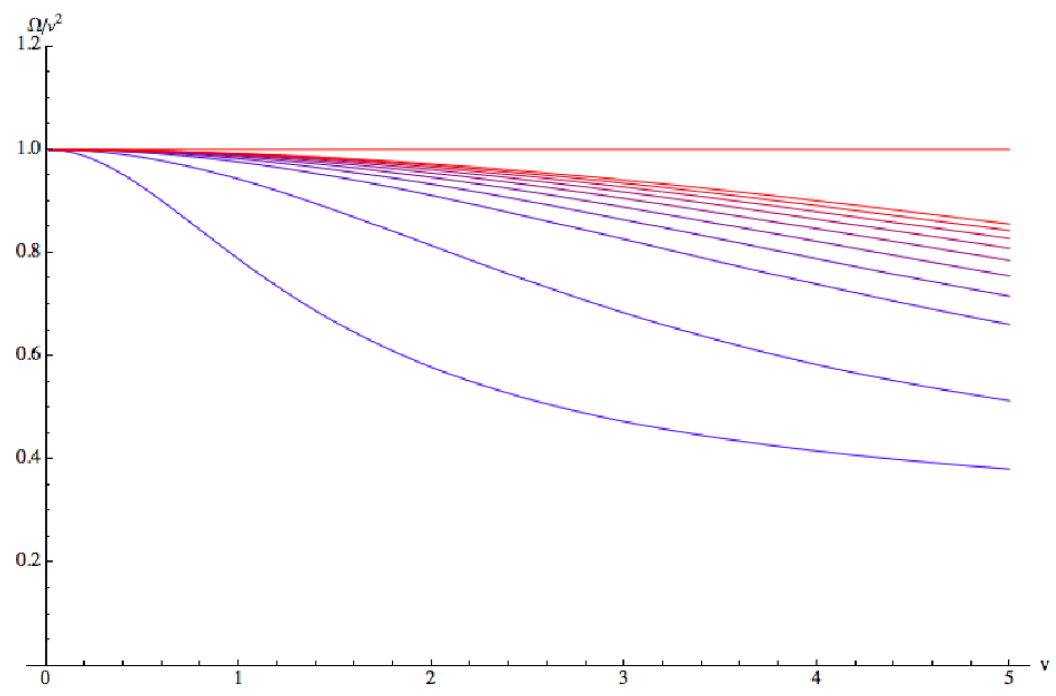

Figure 3. $\Omega / v^{2}$ as a function of $v$ for various RG times. The bottom curve corresponds to the initial, tree-level metric, while the line at $\Omega / v^{2}=1$ corresponds to the flat metric.

In figure 3 we plot the ratio $\Omega / v^{2}$. The asymptotic behavior of the initial metric is $\Omega / v^{2} \approx \frac{1}{4}$, and as RG time increases we see that the behavior at any fixed value of $v$ approaches that of the flat metric, with $\Omega / v^{2}=1$. In figure 4 we plot $\sqrt{g} R$, where $R$ is the Ricci scalar. Here we see that the non-zero curvature is moving outwards from the origin, and spreading out. ${ }^{3}$ Note that the integral of this quantity over $v$ is a topological quantity, and thus it is conserved by the RG flow.

These results indicate that the space near the tip of the cone smooths out with RG time. This flattening modifies the region near the tip to look like flat space. Although the asymptotic behavior is unchanged by the RG flow, the flat region will continue to spread outward, and for any fixed position, the metric near this point will eventually look like the flat metric, for sufficiently long RG times. A similar behavior was observed in [11] in the context of theories with $\mathbb{C} / \mathbb{Z}_{n}$ target space. As argued there, the smoothing of the tip of the cone is consistent with the fact that the RG flow tends to reduce the volume of the target space in the region interior to a given radius.

In this sense, the theory flows in the infrared to the theory of a free chiral multiplet, with target space $\mathcal{M}(\tau=\infty)$ which is the flat complex plane. The IR duality is the claim that if we first flow to the IR, and then compute correlation functions of operators that are localized in the target space, then they will be the same for the gauge theory and for the free theory.

The numerical results above give a good description of the RG flow of the metric only when $|r| \gg 1$, so that the one-loop approximation can be trusted. Nevertheless, we conjecture that this qualitative behavior persists for any non-zero $r$, and all such theories flow to the free theory.

\footnotetext{
${ }^{3}$ The standard intuition that positive curvature flows to larger values and negative curvature flows to smaller values applies in symmetric spaces. This is not the case here and indeed our positive curvature near the origin flows to smaller values.
} 


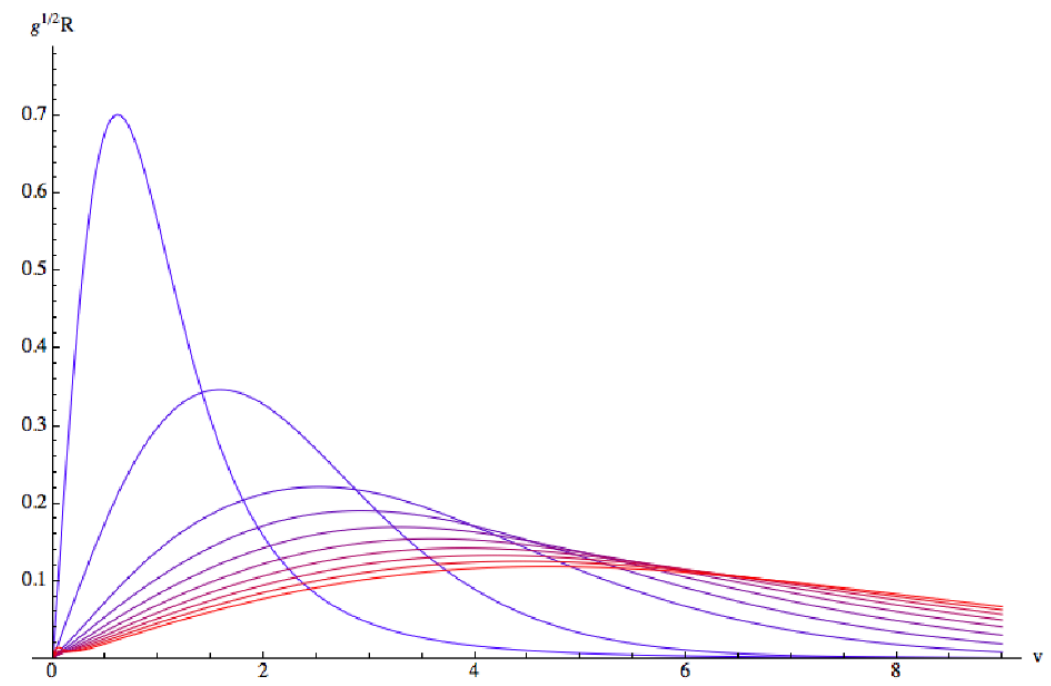

Figure 4. $\sqrt{g} R$ as a function of $v$ for various RG times. The tallest peak corresponds to the initial metric, whose curvature is concentrated near the tip of the cone at $v=0$. As RG time increases, this moves to positive $v$ and spreads outward.

\section{$3 \quad$ Pure $\mathrm{SU}(N)$ gauge theories}

Pure $\mathrm{SU}(N) \mathcal{N}=(2,2)$ gauge theories have a complex scalar $\Sigma$ in the adjoint representation, which can be viewed as the bottom component of a twisted chiral multiplet. They have a classical Coulomb branch of vacua of dimension $N-1$, where the gauge group is broken to $\mathrm{U}(1)^{N-1}$. It may be parameterized by the eigenvalues $\Sigma_{i}$ of $\Sigma$, satisfying $\sum_{i=1}^{N} \Sigma_{i}=0$, which are defined up to permutations (the Weyl group of $\mathrm{SU}(N)$ ). The effective twisted superpotential on this Coulomb branch vanishes, so one expects it to remain also in the quantum theory.

One can obtain an effective theory on the Coulomb branch by integrating out the Wbosons. It is natural to have a kinetic term for $\Sigma$ proportional to $1 / g_{Y M}^{2}$, and then the classical W-boson masses are given by $\left|\Sigma_{i}-\Sigma_{j}\right|$. Classically the metric on the Coulomb branch is conical, with singularities where W-bosons become massless; for instance for $N=2$ it is $\mathbb{C} / \mathbb{Z}_{2}$. Because of the Weyl group identifications on the $\Sigma_{i}$ coordinates, it is convenient to use gauge-invariant coordinates

$$
Y_{n} \equiv \operatorname{tr}\left(\Sigma^{n}\right)=\sum_{i=1}^{N} \Sigma_{i}^{n}
$$

for $n=2,3, \cdots, N$. The classical metric is flat and canonical in the $\Sigma_{i}$ variables

$$
\frac{1}{g_{Y M}^{2}} \sum_{i=1}^{N}\left|d \Sigma_{i}\right|^{2} .
$$

This can be translated into a metric for the $Y_{n}$ variables using (3.1).

Quantum corrections modify (3.2) by a power series in $g_{Y M}^{2} / \Sigma^{2}$ (where $\Sigma$ denotes masses of W-bosons); in canonically normalized dimensionless variables $\tilde{\Sigma}_{i}=\Sigma_{i} / g_{Y M}$, 
they give a power series in $1 / \tilde{\Sigma}^{2}$. The low-energy metric obtained by integrating out the $\mathrm{W}$-bosons is canonical (flat) in the $\Sigma_{i}$ coordinates far from the singularities where W-bosons are massless, but receives large perturbative and non-perturbative corrections there. So we have at energies below $g_{Y M}$ a sigma model with a metric which is a function of $\tilde{\Sigma}_{i}$, or equivalently of the single-valued $Y_{n}$, which we do not know how to compute exactly, though we know its form when all $\left|\Sigma_{i}-\Sigma_{j}\right|$ are large.

We conjecture that:

A. The quantum corrections smooth out the classical singularities, such that the metric becomes smooth everywhere.

B. The resulting sigma model flows under the renormalization group to a flat metric on $\mathbb{C}^{N-1}$ with a canonical form in terms of the gauge-invariant variables $Y_{n}$. Namely, we conjecture that the pure $\mathrm{SU}(N)$ gauge theory is IR-dual to free fields $Y_{n}$, in the sense discussed above. Note that in terms of these variables the original metric is very different from the original canonical metric in terms of $\Sigma_{i}$ even far from the singularities, so this flow requires a change in the asymptotic form of the metric, as in the previous section. For the pure $\mathrm{SU}(2)$ theory, the conjectured change in the asymptotic form of the metric happens to be the same as the one discussed in the previous section.

Since we do not know the original metric of the gauge theory with the quantum corrections, our tests of this conjecture are weaker than in the previous section, and we cannot directly study the RG flow.

One piece of evidence for this conjecture is that the elliptic genus of the pure $\mathrm{SU}(N)$ gauge theory is the same as that of the theory of $(N-1)$ free twisted chiral superfields $Y_{n}$. From [10], the elliptic genus of the pure $\mathrm{SU}(N)$ theory is given by:

$$
\mathcal{Z}_{\mathrm{SU}(N)}^{T^{2}}(q, y)=\frac{\theta\left(y^{-1} ; q\right)}{\theta\left(y^{-N} ; q\right)}
$$

To compare this to the elliptic genus of the $N-1$ free twisted chirals $Y_{n}, n=2, \ldots, N$, note that a free twisted chiral of R-charge $r$ contributes:

$$
\mathcal{Z}_{\text {twisted chiral }}^{T^{2}}(q, y)=\frac{\theta\left(y^{1-r / 2} ; q\right)}{\theta\left(y^{-r / 2} ; q\right)} .
$$

Since $\operatorname{tr}\left(\Sigma^{n}\right)$ has R-charge $2 n$, the collection of these free fields has elliptic genus:

$$
\mathcal{Z}_{\otimes_{n} \operatorname{tr}\left(\Sigma^{n}\right)}^{T^{2}}(q, y)=\prod_{n=2}^{N} \frac{\theta\left(y^{1-n} ; q\right)}{\theta\left(y^{-n} ; q\right)}=\frac{\theta\left(y^{-1} ; q\right)}{\theta\left(y^{-N} ; q\right)},
$$

agreeing with (3.3). Note that the IR theory has an enhanced $\mathrm{U}(N-1)$ flavor symmetry, but since this is not visible in the UV we cannot turn on the corresponding fugacities in the elliptic genus.

For this theory the $\mathbb{S}^{2}$ partition function diverges (there are no twisted chiral parameters that can be used to regularize it), so we cannot use it to test the duality. 
Another important prediction of this scenario, which passes many consistency checks, is the following. Non-Abelian gauge theories with a generic spectrum of massive chiral multiplets have an effective twisted superpotential $\tilde{W}$ on their Coulomb branch, which can be computed exactly at one-loop. The extrema of this superpotential are expected to correspond to supersymmetric vacua. However, sometimes extrema appear at points where some of the $\Sigma_{i}$ are equal, so that some non-Abelian gauge group is classically unbroken (with no massless matter fields); this gauge theory is strongly coupled so the naive analysis based on the effective superpotential cannot be trusted. However, there is a well-defined question of whether there is a supersymmetric vacuum at these points or not. Evidence from the index and other considerations suggests (see, for instance, [5]) that there is actually no supersymmetric vacuum there.

In the scenario above, the effective twisted superpotential near the points of enhanced $\mathrm{SU}(\tilde{N})$ is naturally written in the $\tilde{Y}_{n}$ variables of this effective pure $\mathrm{SU}(\tilde{N})$ theory, which has a smooth metric there. Generically, when expanded near these points, the effective superpotential $\tilde{W}$ will contain terms linear in the $\tilde{Y}_{n}$ (plus higher order terms). This implies that there should not be any supersymmetric vacuum at the point $\tilde{Y}_{2}=\tilde{Y}_{3}=\cdots=0$ of enhanced $\mathrm{SU}(\tilde{N})$, consistent with the known results.

\section{Acknowledgments}

We would like to thank F. Benini and K. Hori for useful discussions. The work of OA and SSR was supported in part by the I-CORE program of the Planning and Budgeting Committee and the Israel Science Foundation (grant number 1937/12). The work of OA was supported in part by an Israel Science Foundation center for excellence grant, by the Minerva foundation with funding from the Federal German Ministry for Education and Research, by a Henri Gutwirth award from the Henri Gutwirth Fund for the Promotion of Research, and by the ISF within the ISF-UGC joint research program framework (grant no. 1200/14). OA is the Samuel Sebba Professorial Chair of Pure and Applied Physics. SSR is a Jacques Lewiner Career Advancement Chair fellow. The research of SSR was also supported by the Israel Science Foundation under grant no. 1696/15. NS was supported in part by DOE grant DE-SC0009988. NS thanks the hospitality of the Weizmann Institute of Science during the completion of this work. BW was supported in part by the National Science Foundation under Grant No. NSF PHY11-25915.

Open Access. This article is distributed under the terms of the Creative Commons Attribution License (CC-BY 4.0), which permits any use, distribution and reproduction in any medium, provided the original author(s) and source are credited.

\section{References}

[1] O. Aharony, S. Razamat, N. Seiberg and B. Willett, work in progress.

[2] E. Witten, Phases of $N=2$ theories in two-dimensions, Nucl. Phys. B 403 (1993) 159 [hep-th/9301042] [INSPIRE]. 
[3] K. Hori and A. Kapustin, Duality of the fermionic 2-D black hole and $N=2$ Liouville theory as mirror symmetry, JHEP 08 (2001) 045 [hep-th/0104202] [INSPIRE].

[4] O. Aharony, A. Feldman and B. Willett, work in progress.

[5] K. Hori and D. Tong, Aspects of Non-Abelian Gauge Dynamics in Two-Dimensional $N=(2,2)$ Theories, JHEP 05 (2007) 079 [hep-th/0609032] [INSPIRE].

[6] K. Hori, Duality In Two-Dimensional (2,2) Supersymmetric Non-Abelian Gauge Theories, JHEP 10 (2013) 121 [arXiv:1104.2853] [INSPIRE].

[7] F. Benini and S. Cremonesi, Partition Functions of $\mathcal{N}=(2,2)$ Gauge Theories on $S^{2}$ and Vortices, Commun. Math. Phys. 334 (2015) 1483 [arXiv:1206.2356] [InSPIRE].

[8] N. Doroud, J. Gomis, B. Le Floch and S. Lee, Exact Results in D $=2$ Supersymmetric Gauge Theories, JHEP 05 (2013) 093 [arXiv:1206.2606] [INSPIRE].

[9] F. Benini, D.S. Park and P. Zhao, Cluster Algebras from Dualities of $2 d \mathcal{N}=(2,2)$ Quiver Gauge Theories, Commun. Math. Phys. 340 (2015) 47 [arXiv:1406.2699] [InSPIRE].

[10] F. Benini, R. Eager, K. Hori and Y. Tachikawa, Elliptic Genera of $2 d \mathcal{N}=2$ Gauge Theories, Commun. Math. Phys. 333 (2015) 1241 [arXiv:1308.4896] [INSPIRE].

[11] A. Adams, J. Polchinski and E. Silverstein, Don't panic! Closed string tachyons in ALE space-times, JHEP 10 (2001) 029 [hep-th/0108075] [INSPIRE].

[12] J.A. Harvey, D. Kutasov, E.J. Martinec and G.W. Moore, Localized tachyons and RG flows, hep-th/0111154 [INSPIRE].

[13] M. Gutperle, M. Headrick, S. Minwalla and V. Schomerus, Space-time energy decreases under world sheet RG flow, JHEP 01 (2003) 073 [hep-th/0211063] [INSPIRE].

[14] E.J. Martinec and G.W. Moore, On decay of k-theory, hep-th/0212059 [INSPIRE].

[15] C. Vafa, Mirror symmetry and closed string tachyon condensation, in "From fields to strings", M. Shifman (ed.) et al., vol. 3, p. 1828 [hep-th/0111051] [INSPIRE].

[16] I. Bakas, Renormalization group flows and continual Lie algebras, JHEP 08 (2003) 013 [hep-th/0307154] [INSPIRE].

[17] T. Oliynyk, V. Suneeta and E. Woolgar, Irreversibility of world-sheet renormalization group flow, Phys. Lett. B 610 (2005) 115 [hep-th/0410001] [InSPIRE].

[18] I. Bakas, Ricci flows and their integrability in two dimensions, Comptes Rendus Physique 6 (2005) 175 [hep-th/0410093] [INSPIRE].

[19] F. Benini, R. Eager, K. Hori and Y. Tachikawa, Elliptic genera of two-dimensional $N=2$ gauge theories with rank-one gauge groups, Lett. Math. Phys. 104 (2014) 465 [arXiv: 1305.0533] [INSPIRE]. 